\title{
Breath Carbon Monoxide Monitor
}

National Cancer Institute

\section{Source}

National Cancer Institute. Breath Carbon Monoxide Monitor. NCI Thesaurus. Code C139923.

A device designed to measure carbon monoxide in a smoker's breath as a means toward smoking cessation. 